\title{
Finite element approximation of a system coupling curve evolution with prescribed normal contact to a fixed boundary to reaction-diffusion on the curve
}

Vanessa Styles and James Van Yperen

\begin{abstract}
We consider a finite element approximation for a system consisting of the evolution of a curve evolving by forced curve shortening flow coupled to a reactiondiffusion equation on the evolving curve. The curve evolves inside a given domain $\Omega \subset \mathbb{R}^{2}$ and meets $\partial \Omega$ orthogonally. The scheme for the coupled system is based on the schemes derived in [1] and [5]. We present numerical experiments and show the experimental order of convergence of the approximation.
\end{abstract}

\section{Introduction}

We consider a curve $\Gamma(t)$ evolving by forced curve shortening flow inside a given bounded domain $\Omega \subset \mathbb{R}^{2}$, with the forcing being a function of the solution, $w: \Gamma(t) \rightarrow \mathbb{R}$, of a reaction-diffusion equation that holds on $\Gamma(t)$, such that

$$
\begin{aligned}
v & =\kappa+f(w) & & \text { on } \Gamma(t), t \in(0, T], \\
\partial_{t}^{\bullet} w & =w_{s s}+\kappa v w+g(v, w) & & \text { on } \Gamma(t), t \in(0, T],
\end{aligned}
$$

subject to the initial data $\Gamma(0)=\Gamma_{0}$ and $w(\cdot, 0)=w_{0}$ on $\Gamma_{0}$.

Here $v$ and $\kappa$ respectively denote the normal velocity and mean curvature of $\Gamma(t)$, corresponding to the choice $\mathbf{n}$ of a unit normal, $s$ is the arclength parameter on $\Gamma(t)$ and $\partial_{t}^{\bullet} w:=w_{t}+v \frac{\partial w}{\partial \mathbf{n}}$ denotes the material derivative of $w$. In addition we impose that the curve meets the boundary $\partial \Omega$ orthogonally. To this end we assume that $\partial \Omega$ is given by a smooth function $F$ such that

$$
\partial \Omega=\left\{p \in \mathbb{R}^{2}: F(p)=0\right\} \text { and }|\nabla F(p)|=1 \forall p \in \partial \Omega .
$$

Vanessa Styles

Department of Mathematics, University of Sussex, e-mail: v.styles@sussex.ac.uk

James Van Yperen

Department of Mathematics, University of Sussex, e-mail: j.vanyperen@sussex.ac.uk 
Coupling the parametrisation of (1), (2) that is presented in [1] for the setting in which $\Gamma(t)$ is a closed curve, with the formulation of (1) presented in [5] for the setting in which $\Gamma(t)$ meets the boundary $\partial \Omega$ orthogonally, yields the following system:

$$
\begin{array}{lr}
\alpha \mathbf{x}_{t}+(1-\alpha)\left(\mathbf{x}_{t} \cdot \mathbf{n}\right) \mathbf{n}=\frac{\mathbf{x}_{\rho \rho}}{\left|\mathbf{x}_{\rho}\right|^{2}}+f(w) \mathbf{n}, & \text { for }(\rho, t) \in \mathbb{I} \times(0, T) \\
w_{t}-\left(\mathbf{x}_{t} \cdot \tau\right) \frac{1}{\left|\mathbf{x}_{\rho}\right|} w_{\rho}-\frac{1}{\left|\mathbf{x}_{\rho}\right|}\left(\frac{w_{\rho}}{\left|\mathbf{x}_{\rho}\right|}\right)_{\rho}-\kappa v w=g(v, w), & \text { for }(\rho, t) \in \mathbb{I} \times(0, T) \\
\mathbf{x}(\rho, 0)=\mathbf{x}_{0}(\rho), \quad w(\rho, 0)=w_{0}(\rho) & \text { for } \rho \in \mathbb{I} \\
w(\rho, t)=w_{b}, & \text { for }(\rho, t) \in\{0,1\} \times[0, T] \\
F(\mathbf{x}(\rho, t))=0, & \text { for }(\rho, t) \in\{0,1\} \times[0, T] \\
\mathbf{x}_{\rho}(\rho, t) \cdot \nabla^{\perp} F(\mathbf{x}(\rho, t)=0, & \text { for }(\rho, t) \in\{0,1\} \times[0, T] .
\end{array}
$$

Here $\alpha \in(0,1], \mathbb{I}:=(0,1), \mathbf{x}(\cdot, t):[0,1] \rightarrow \mathbb{R}^{2}, w(\rho, t):=w(\mathbf{x}(\rho, t), t),(\rho, t) \in$ $[0,1] \times[0, T]$, and the unit tangent and unit normal to $\Gamma(t)$ are respectively given by $\boldsymbol{\tau}=\mathbf{x}_{s}=\frac{\mathbf{x}_{\rho}}{\left|\mathbf{x}_{\rho}\right|}$ and $\mathbf{n}=\tau^{\perp}$ where $(\cdot)^{\perp}$ denotes counter-clockwise rotation by $\frac{\pi}{2}$.

The formulation of curve shortening flow in the form of (3) for a closed curve in $\mathbb{R}^{2}$ was presented and analysed in [8], where the DeTurck trick is used in coupling the motion of the curve to the harmonic map heat flow, with the parameter $\alpha \in(0,1]$ being such that $1 / \alpha$ corresponds to the diffusion coefficient in the harmonic map heat flow. Setting $\alpha \in(0,1]$ introduces a tangential part in the velocity which, at the numerical level, gives rise to a good distribution of the mesh points along the curve. Setting $\alpha=1$, one recovers the formulation introduced and analysed in [4], while formally setting $\alpha=0$ yields the approach introduced in [3]. In [6] the authors derive finite element approximations of a simplified version of the parametric coupled system (3)-(8), and two related models. In particular, the evolution law for the parametric system derived in [6] can be obtained from (3) by setting $\alpha=1, F(x, y)=|x|-1$ and considering a slightly different formulation of the reaction-diffusion equation (4). In [1] the authors prove optimal error bounds for a fully discrete finite element approximation of the coupled system (3)-(5) for the case where $\Gamma(t)$ is a closed curve in $\mathbb{R}^{2}$. While in [9] optimal error bounds are presented for a semi-discrete finite element approximation of an alternative formulation, which is introduced and analysed in [7], of the coupled system (3)-(5), for the case where $\Gamma(t)$ is a closed curve in $\mathbb{R}^{2}$. Setting $\alpha=1$ and $f(w)=0$ in (3) and coupling the resulting equation to (7), (8) gives rise to the model presented and analysed in [5], in which optimal order error bounds for a semi-discrete finite element approximation of curve shortening flow with a prescribed normal contact to a fixed boundary are presented. In [2] the authors propose parametric finite element approximations of combined second and fourth order geometric evolution equations for curves that are connected via triple or quadruple junctions or that intersect external boundaries. 


\section{Weak formulation and finite element approximation}

For a weak formulation of (3) we multiply it by $\left|\mathbf{x}_{\rho}\right|^{2} \boldsymbol{\xi}$, where $\xi \in\left[H^{1}(\mathbb{I})\right]^{2}$ is a test function, integrate in space, use integration by parts and (8) to obtain

$$
\begin{aligned}
& \left(\left|\mathbf{x}_{\rho}\right|^{2}\left[\alpha \mathbf{x}_{t}+(1-\alpha)\left(\mathbf{x}_{t} \cdot \mathbf{n}\right) \mathbf{n}\right], \boldsymbol{\xi}\right)+\left(\mathbf{x}_{\rho}, \boldsymbol{\xi}_{\rho}\right) \\
& \quad=\left[\left(\mathbf{x}_{\rho} \cdot \nabla F(\mathbf{x})\right)(\boldsymbol{\xi} \cdot \nabla F(\mathbf{x}))\right]_{0}^{1}+\left(\left|\mathbf{x}_{\rho}\right|^{2} f(w) \mathbf{n}, \boldsymbol{\xi}\right) \quad \forall \boldsymbol{\xi} \in\left[H^{1}(\mathbb{I})\right]^{2},
\end{aligned}
$$

where $(\cdot, \cdot)$ denotes the standard $L^{2}(\mathbb{I})$ inner product. For a weak formulation of (4) we multiply it by $\left|\mathbf{x}_{\rho}\right| \eta$, where $\eta \in H_{0}^{1}(\mathbb{I})$ is a time-independent test function, integrate in space, use integration by parts and note that $\tau_{\rho}=\kappa \mathbf{n}\left|\mathbf{x}_{\rho}\right|$ to obtain

$$
\frac{d}{d t}\left(\left|\mathbf{x}_{\rho}\right| w, \eta\right)+\left(\psi w, \eta_{\rho}\right)+\left(\frac{w_{\rho}}{\left|\mathbf{x}_{\rho}\right|}, \eta_{\rho}\right)=\left(\left|\mathbf{x}_{\rho}\right| g(v, w), \eta\right) \quad \forall \eta \in H_{0}^{1}(\mathbb{I}) .
$$

Here $\psi$ denotes the tangential velocity of $\Gamma(t)$, whereby the normal and tangential velocities of $\Gamma(t)$ are given by $v=\mathbf{x}_{t} \cdot \mathbf{n}$ and $\psi=\mathbf{x}_{t} \cdot \tau$. We now introduce a finite element approximation of (9), (10). We first let $0=t_{0}<t_{1}<\cdots<t_{N-1}<t_{N}=T$ be a partition of $[0, T]$ with $\Delta t_{n}:=t_{n}-t_{n-1}$. Next we partition the interval $\mathbb{I}$ such that $\mathbb{I}=\cup_{j=1}^{J} \overline{\sigma_{j}}$, where $\sigma_{j}=\left(\rho_{j-1}, \rho_{j}\right)$, with $h_{j}=\rho_{j}-\rho_{j-1}$. We set

$$
\begin{aligned}
& V^{h}:=\left\{\chi \in C(\mathbb{I}): \chi_{\left.\right|_{j}} \text { is affine, } j=1, \ldots, J\right\} \subset H^{1}(\mathbb{I}) \\
& V_{0}^{h}:=\left\{\chi \in V^{h}: \chi\left(\rho_{j}\right)=0, \text { for } j \in\{0, J\}\right\}
\end{aligned}
$$

and denote the standard Lagrange interpolation operator by $I^{h}: C(\mathbb{I}) \rightarrow V^{h}$, where $\left(I^{h} \eta\right)\left(\rho_{j}\right)=\eta\left(\rho_{j}\right)$, for $j=1, \ldots, J$. We define the discrete inner product $\left(\eta_{1}, \eta_{2}\right)^{h}$ by

$$
\left(\eta_{1}, \eta_{2}\right)^{h}:=\sum_{j=1}^{J} \int_{\sigma_{j}} I_{j}^{h}\left(\eta_{1} \eta_{2}\right) \mathrm{d} \rho,
$$

where $I_{j}^{h}=I_{\left.\right|_{\sigma_{j}}}^{h}$ is the local interpolation operator. Our finite element approximation of (9), (10) then takes the form:

Given $\left(\mathbf{X}^{n-1}, W^{n-1}-w_{b}\right) \in\left[V^{h}\right]^{2} \times V_{0}^{h}$, find $\left(\mathbf{X}^{n}, W^{n}-w_{b}\right) \in\left[V^{h}\right]^{2} \times V_{0}^{h}$ such that for all $\left(\xi^{h}, \eta^{h}\right) \in\left[V^{h}\right]^{2} \times V_{0}^{h}$ we have

$$
\begin{gathered}
\left(\left|\mathbf{X}_{\rho}^{n-1}\right|^{2}\left[\alpha D_{t} \mathbf{X}^{n}+(1-\alpha)\left(D_{t} \mathbf{X}^{n} \cdot \mathcal{N}^{n-1}\right) \mathcal{N}^{n-1}\right], \xi^{h}\right)^{h}+\left(\mathbf{X}_{\rho}^{n}, \boldsymbol{\xi}_{\rho}^{h}\right) \\
=\left[\left(\mathbf{X}_{\rho}^{n} \cdot \nabla F\left(\mathbf{X}^{n}\right)\right)\left(\boldsymbol{\xi}^{h} \cdot \nabla F\left(\mathbf{X}^{n}\right)\right)\right]_{0}^{1}+\left(\left|\mathbf{X}_{\rho}^{n-1}\right|^{2} f\left(W^{n-1}\right) \mathcal{N}^{n-1}, \boldsymbol{\xi}^{h}\right)^{h} \\
D_{t}\left[\left(\left|\mathbf{X}_{\rho}^{n}\right| W^{n}, \eta^{h}\right)^{h}\right]+\left(\frac{W_{\rho}^{n}}{\left|\mathbf{X}_{\rho}^{n}\right|}, \eta_{\rho}^{h}\right)+\left(\Psi^{n} W^{n}, \eta_{\rho}^{h}\right)^{h} \\
=\left(\left|\mathbf{X}_{\rho}^{n}\right| g\left(V^{n}, W^{n-1}\right), \eta^{h}\right)^{h}
\end{gathered}
$$


with the additional boundary constraint

$$
F\left(\mathbf{X}_{0}^{n}\right)=F\left(\mathbf{X}_{J}^{n}\right)=0 .
$$

Here and in what follows we set $D_{t}\left(a^{n}\right):=\left(a^{n}-a^{n-1}\right) / \Delta t_{n}$ and on $\sigma_{j}, j=1, \ldots, J$ we set $\mathcal{T}^{n}=\frac{\mathbf{X}_{\rho}^{n}}{\left|\mathbf{X}_{\rho}^{n}\right|}, \mathcal{N}^{n}=\left(\mathcal{T}^{n}\right)^{\perp}, \Psi^{n}=D_{t} \mathbf{X}^{n} \cdot \mathcal{T}^{n}$ and $V^{n}=D_{t} \mathbf{X}^{n} \cdot \mathcal{N}^{n}$.

Remark 1 In [5], rather than use the nonlinear scheme presented above to approximate (3), (7), (8), the authors present a linear scheme in which (7) is not necessarily satisfied but is instead approximated through the relation $0=\frac{d}{d t} F(\mathbf{x})=\mathbf{x}_{t} \cdot \nabla F(\mathbf{x})$.

\section{Numerical results}

\subsection{Solution of the discrete system (11), (13)}

We solve the resulting system of nonlinear algebraic equations arising at each time level from the approximation (11), (13), with $\xi^{h}=\chi_{j}, j=1, \ldots, J-1, \xi^{h}=$ $\nabla^{\perp} F\left(\mathbf{X}^{n}\right) \chi_{0}$ and $\xi^{h}=\nabla^{\perp} F\left(\mathbf{X}^{n}\right) \chi_{J}$, using the following Newton scheme, where for ease of presentation we set $\alpha=1$ and $f(w)=0$ :

Given $\mathbf{X}^{n, i-1}$, with $\mathbf{X}^{n, 0}=\mathbf{X}^{n-1}$, we set $\mathbf{X}^{n, i}:=\mathbf{X}^{n, i-1}+\delta^{i}$ such that for $j=$ $1, \ldots, J-1, \boldsymbol{\delta}^{i}$ solves

$$
\begin{aligned}
& \frac{1}{2}\left(q_{j}^{n-1}+q_{j-1}^{n-1}\right) \frac{\boldsymbol{\delta}_{j}^{i}}{\Delta t_{n}}-\left(\boldsymbol{\delta}_{j-1}^{i}-2 \delta_{j}^{i}+\boldsymbol{\delta}_{j+1}^{i}\right) \\
& \quad=-\frac{1}{2}\left(q_{j}^{n-1}+q_{j-1}^{n-1}\right) D_{t}\left(\mathbf{X}_{j}^{n, i-1}\right)+\left(\mathbf{X}_{j-1}^{n, i-1}-2 \mathbf{X}_{j}^{n, i-1}+\mathbf{X}_{j+1}^{n, i-1}\right) \\
& \frac{1}{2} q_{0}^{n-1}\left[\left(\frac{\delta_{0}^{i}}{\Delta t_{n}} \cdot \nabla^{\perp} F\left(\mathbf{X}_{0}^{n, i-1}\right)\right)+\left(D_{t}\left(\mathbf{X}_{0}^{n, i-1}\right) \cdot D_{\perp}^{2} F\left(\mathbf{X}_{0}^{n, i-1}\right) \boldsymbol{\delta}_{0}^{i}\right)\right] \\
& \quad-\left(\left(\boldsymbol{\delta}_{1}^{i}-\boldsymbol{\delta}_{0}^{i}\right) \cdot \nabla^{\perp} F\left(\mathbf{X}_{0}^{n, i-1}\right)\right)-\left(\left(\mathbf{X}_{1}^{n, i-1}-\mathbf{X}_{0}^{n, i-1}\right) \cdot D_{\perp}^{2} F\left(\mathbf{X}_{0}^{n, i-1}\right) \boldsymbol{\delta}_{0}^{i}\right) \\
& \quad=-\frac{1}{2} q_{0}^{n-1}\left(D_{t}\left(\mathbf{X}_{0}^{n, i-1}\right) \cdot \nabla^{\perp} F\left(\mathbf{X}_{0}^{n, i-1}\right)\right)+\left(\left(\mathbf{X}_{1}^{n, i-1}-\mathbf{X}_{0}^{n, i-1}\right) \cdot \nabla^{\perp} F\left(\mathbf{X}_{0}^{n, i-1}\right)\right) \\
& \frac{1}{2} q_{J-1}^{n-1}\left[\left(\frac{\delta_{J}^{i}}{\Delta t_{n}} \cdot \nabla^{\perp} F\left(\mathbf{X}_{J}^{n, i-1}\right)\right)+\left(D_{t}\left(\mathbf{X}_{J}^{n, i-1}\right) \cdot D_{\perp}^{2} F\left(\mathbf{X}_{J}^{n, i-1}\right) \boldsymbol{\delta}_{J}^{i}\right)\right] \\
& \quad-\left(\left(\boldsymbol{\delta}_{J-1}^{i}-\boldsymbol{\delta}_{J}^{i}\right) \cdot \nabla^{\perp} F\left(\mathbf{X}_{J}^{n, i-1}\right)\right)-\left(\left(\mathbf{X}_{J-1}^{n, i-1}-\mathbf{X}_{J}^{n, i-1}\right) \cdot D_{\perp}^{2} F\left(\mathbf{X}_{J}^{n, i-1}\right) \boldsymbol{\delta}_{J}^{i}\right) \\
& \quad=-\frac{1}{2} q_{J-1}^{n-1}\left(D_{t}\left(\mathbf{X}_{J}^{n, i-1}\right) \cdot \nabla^{\perp} F\left(\mathbf{X}_{J}^{n, i-1}\right)\right)+\left(\left(\mathbf{X}_{J-1}^{n, i-1}-\mathbf{X}_{J}^{n, i-1}\right) \cdot \nabla^{\perp} F\left(\mathbf{X}_{J}^{n, i-1}\right)\right) \\
& \left(\nabla F\left(\mathbf{X}_{0}^{n, i-1}\right) \cdot \boldsymbol{\delta}_{0}^{i}\right)=-F\left(\mathbf{X}_{0}^{n, i-1}\right) \text { and }\left(\nabla F\left(\mathbf{X}_{J}^{n, i-1}\right) \cdot \boldsymbol{\delta}_{J}^{i}\right)=-F\left(\mathbf{X}_{J}^{n, i-1}\right)
\end{aligned}
$$


where $q_{j}^{n-1}=\left|\mathbf{X}_{j+1}^{n-1}-\mathbf{X}_{j}^{n-1}\right|^{2}, D_{\perp}^{2}=\left(\begin{array}{cc}-\partial_{x y} & -\partial_{y y} \\ \partial_{x x} & \partial_{x y}\end{array}\right)$, and in an abuse of notation we have redefined $D_{t}$ from the previous section such that $D_{t}\left(\mathbf{X}_{j}^{n, i-1}\right):=$ $\left(\mathbf{X}_{j}^{n, i-1}-\mathbf{X}_{j}^{n-1}\right) / \Delta t_{n}$. We adopt the stopping criteria $\max _{j=0, J}\left|F\left(\mathbf{X}_{j}^{n, i}\right)\right| \leq \tau$ for some predetermined tolerance, $\tau$.

\subsection{Experimental order of convergence of (11), (13)}

We investigate the experimental order of convergence of (11), (13) by monitoring the following errors:

$$
\mathcal{E}_{1}:=\sup _{n=0, \ldots, N}\left\|I^{h}\left(\mathbf{x}_{\rho}^{n}\right)-\mathbf{X}_{\rho}^{n}\right\|_{\left[L^{2}(\mathbb{I})\right]^{2}}^{2}, \quad \mathcal{E}_{2}:=\sum_{n=1}^{N} \Delta t_{n}\left\|D_{t}\left(I^{h}\left(\mathbf{x}^{n}\right)-\mathbf{X}^{n}\right)\right\|_{\left[L^{2}(\mathbb{I})\right]^{2}}^{2} .
$$

In addition we show how the choice of $\alpha$ affects the size of the errors. In all examples we use a uniform mesh size $h J=1$ and a uniform time step size $\Delta t=h^{2}$.

Example 1: In the first example we set $T=0.4$ and $\Omega:=\mathbb{R} \times \mathbb{R}_{>0}$, such that $\partial \Omega$ is given by $F(x, y)=y$. Taking $\Gamma(0)$ to be a semi circle with radius 1 , the explicit solution is given by

$$
\mathbf{x}(\rho, t)=\sqrt{1-2 t}(\cos (\pi \rho), \sin (\pi \rho))^{T} .
$$

In the left-hand plot in Figure 1 we display: $\mathbf{X}^{0}$ in black, $\mathbf{X}^{n}$ at $t^{n}=0.08 k, k=$ $1, \ldots, 5$, in blue, and $\partial \Omega$ in red, while in Table 1 we display the values of $\mathcal{E}_{i}, i=1,2$, for $\alpha=1$ (left) and $\alpha=0.5$ (right). For both values of $\alpha$ we see eocs close to four, however we note that the errors for $\alpha=0.5$ are significantly smaller than those for $\alpha=1$.

Table 1 Errors and eocs for the shrinking semi circle with $\alpha=1$ (left) and $\alpha=0.5$ (right).

\begin{tabular}{llllll}
\hline$J$ & $N$ & $\mathcal{E}_{1} \times e^{-3}$ & eoc $_{1}$ & $\mathcal{E}_{2} \times e^{-4}$ & eoc $_{2}$ \\
\hline 10 & 40 & 4.672 & - & 20.16 & - \\
20 & 160 & 0.3997 & 3.55 & 1.859 & 3.44 \\
40 & 640 & 0.02726 & 3.87 & 0.1298 & 3.84 \\
80 & 2560 & 0.001742 & 3.97 & 0.008347 & 3.96 \\
\hline
\end{tabular}

\begin{tabular}{llllll}
\hline$J$ & $N$ & $\mathcal{E}_{1} \times e^{-3}$ & eoc $_{1}$ & $\mathcal{E}_{2} \times e^{-4}$ & eoc $_{2}$ \\
\hline 10 & 40 & 1.589 & - & 8.884 & - \\
20 & 160 & 0.1389 & 3.52 & 0.8302 & 3.42 \\
40 & 640 & 0.009514 & 3.87 & 0.05798 & 3.84 \\
80 & 2560 & 0.0006087 & 3.97 & 0.003729 & 3.96 \\
\hline
\end{tabular}

Example 2: In the second example we set $T=0.5$ and $\Omega$ to be the unit disc with centre $(0,0)$, such that $\partial \Omega$ is given by $F(x, y)=\frac{1}{2}\left(x^{2}+y^{2}-1\right)$. In contrast to the previous example this example has been constructed so that $|\nabla F(p)|=1$ is only satisfied on $\partial \Omega$. By setting $f(\rho, t)=\frac{4\left(\rho-\frac{1}{2}\right)}{(1-2 t)^{2}+1}$ the explicit solution is given by 

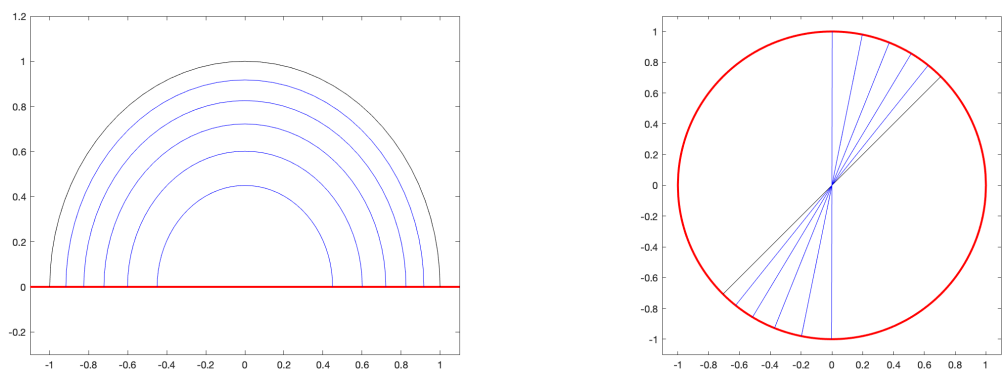

Fig. $1 \mathrm{X}^{n}$ at $t^{n}=0,0.08,0.16,0.24,0.32,0.4$ for the shrinking semi-circle (left), and $t^{n}=$ $0,0.1,0.2,0.3,0.4,0.5$ for the rotating diameter (right).

Table 2 Errors and eocs for the rotating diameter with $\alpha=1$ (left) and $\alpha=0.5$ (right).

\begin{tabular}{llllll}
\hline$J$ & $N$ & $\mathcal{E}_{1} \times e^{-4}$ & $e^{o c_{1}}$ & $\mathcal{E}_{2} \times e^{-5}$ & eoc $_{2}$ \\
\hline 10 & 50 & 1.440 & - & 3.040 & - \\
20 & 200 & 0.09198 & 3.97 & 0.1925 & 3.98 \\
40 & 800 & 0.005780 & 3.99 & 0.01207 & 4.00 \\
80 & 3200 & 0.0003617 & 4.00 & 0.0007552 & 4.00 \\
\hline
\end{tabular}

\begin{tabular}{llllll}
\hline$J$ & $N$ & $\mathcal{E}_{1} \times e^{-4}$ & eoc $_{1}$ & $\mathcal{E}_{2} \times e^{-5}$ & eoc $_{2}$ \\
\hline 10 & 50 & 1.181 & - & 2.710 & - \\
20 & 200 & 0.07459 & 3.98 & 0.1716 & 3.98 \\
40 & 800 & 0.004674 & 4.00 & 0.01076 & 4.00 \\
80 & 3200 & 0.0002923 & 4.00 & 0.0006727 & 4.00 \\
\hline
\end{tabular}

$$
\mathbf{x}(\rho, t)=\frac{2\left(\rho-\frac{1}{2}\right)}{\sqrt{(1-2 t)^{2}+1}}(1-2 t, 1)^{T},
$$

such that $\Gamma(t)$ is a rotating straight line that spans the diameter of $\Omega$. In the right-hand plot of Figure 1 we display: $\mathbf{X}^{0}$ in black, $\mathbf{X}^{n}$ at $t^{n}=0.1 k, k=1, \ldots, 5$, in blue, and $\partial \Omega$ in red, while Table 2 displays the errors $\mathcal{E}_{i}, i=1,2$, for $\alpha=1$ (left) and $\alpha=0.5$ (right). As in Example 1, both values of $\alpha$ exhibit eocs close to four, with the errors obtained using $\alpha=0.5$ being smaller than those obtain using $\alpha=1$. However the difference in the errors for the two values of $\alpha$ in this example is much smaller than the difference in the errors for the two values of $\alpha$ in Example 1, we believe that this is due to the fact that in this example $\mathbf{x}$ is a linear function.

To demonstrate Remark 1 in Section 2, we include Table 3 in which we display errors obtained using the scheme in [5]. In particular we display $\mathcal{E}_{i}, i=1,2,3$, with

$$
\mathcal{E}_{3}:=\sup _{n=0, \ldots, N} \sup _{j=0, \cdots, J}\left|F\left(\mathbf{X}_{j}^{n}\right)\right| \text {. }
$$

Comparing the errors in Tables 2 and 3 we see that the magnitude of the errors for the Newton scheme, (14a)-(14d), are significantly smaller than the errors for the linear scheme in [5]. 
Table 3 Errors and eocs for the rotating diameter for scheme proposed in [5].

\begin{tabular}{llllllll}
\hline$J$ & $N$ & $\mathcal{E}_{1} \times e^{-4}$ & eoc $_{1}$ & $\mathcal{E}_{2} \times e^{-5}$ & eoc $_{2}$ & $\mathcal{E}_{3} \times e^{-3}$ & eoc $_{3}$ \\
\hline 10 & 50 & 43.83 & & 76.20 & & 5.771 & \\
20 & 200 & 3.175 & 3.79 & 5.442 & 3.81 & 1.563 & 0.94 \\
40 & 800 & 0.2076 & 3.93 & 0.3542 & 3.94 & 0.3989 & 0.99 \\
80 & 3200 & 0.01317 & 3.98 & 0.02243 & 3.98 & 0.1003 & 1.00 \\
\hline
\end{tabular}

\subsection{Experimental order of convergence of the coupled scheme (11)-(13)}

We conclude our numerical results by investigating the experimental order of convergence of the coupled scheme (11)-(13). In addition to monitoring the errors $\mathcal{E}_{i}$, $i=1,2$, we also monitor

$$
\mathcal{E}_{4}:=\sup _{n=0, \ldots, N}\left\|I^{h}\left(w^{n}\right)-W^{n}\right\|_{L^{2}(\mathbb{I})}^{2}, \quad \mathcal{E}_{5}:=\sum_{n=1}^{n} \Delta t_{n}\left\|I^{h}\left(w_{\rho}^{n}\right)-W_{\rho}^{n}\right\|_{L^{2}(\mathbb{I})}^{2} .
$$

We adopt the same set-up as in Example 2, with $T=0.5$ and $\Omega$ being the unit disc with centre $(0,0)$, such that $\partial \Omega$ is given by $F(x, y)=\frac{1}{2}\left(x^{2}+y^{2}-1\right)$. Setting $f(\rho, t)=\frac{4\left(\rho^{2}-\frac{w(\rho, t)}{1-t}-\frac{1}{2}\right)}{(1-2 t)^{2}+1}$ and $g=\frac{t-1}{2}-\frac{w(\rho, t)}{1-t}$, the explicit solution is given by

$$
x=\frac{2\left(\rho-\frac{1}{2}\right)}{\sqrt{(1-2 t)^{2}+1}}(1-2 t, 1)^{T} \text { and } w=(1-t) \rho(\rho-1),
$$

such that $w$ describes a shrinking parabola and, as in Example 2, $\Gamma(t)$ is a rotating straight line that spans the diameter of $\Omega$. In the left-hand plot of Figure 2 we display: $\mathbf{X}^{0}$ in black, $\mathbf{X}^{n}$, at $t^{n}=0.1 k, k=1, \ldots, 5$, in blue, and $\partial \Omega$ in red, while in the right-hand plot we display: $W^{0}$ in black and $W^{n}$, at $t^{n}=0.1 k, k=1, \ldots, 5$ in blue. In Table 4 we present the experimental order of convergence for the errors obtained using $\alpha=0.5$, we do not present the errors for $\alpha=1$ since they are very similar to those obtained using $\alpha=0.5$. For all the four errors we see eocs close to four.

Remark 2 In the three examples presented above if we take $\Delta t=C h$ we observe eocs close to two rather than the eocs close to four that we observe above for $\Delta t=C h^{2}$. Similar convergence behaviour was observed in [1].

Acknowledgements JVY gratefully acknowledges the support of the EPSRC grant 1805391. VS would like to thank the Isaac Newton Institute for Mathematical Sciences for support and hospitality during the programme Geometry, compatibility and structure preservation in computational differential equations when work on this paper was undertaken. This work was supported by: EPSRC grant number EP/R014604/1. 
Table 4 Errors and eocs for the parabola defined on the rotating diameter with $\alpha=0.5$.

\begin{tabular}{llllllllll}
\hline$J$ & $N$ & $\mathcal{E}_{1} \times e^{-4}$ & $e^{o c_{1}}$ & $\mathcal{E}_{2} \times e^{-5}$ & eoc $_{2}$ & $\mathcal{E}_{4} \times e^{-6}$ & eoc $_{4}$ & $\mathcal{E}_{5} \times e^{-6}$ & eoc $_{5}$ \\
\hline 10 & 50 & 1.205 & - & 3.756 & - & 1.207 & - & 3.073 & - \\
20 & 200 & 0.07643 & 3.98 & 0.2453 & 3.94 & 0.07829 & 3.95 & 0.2010 & 3.93 \\
40 & 800 & 0.004795 & 3.99 & 0.01551 & 3.98 & 0.004937 & 3.99 & 0.01271 & 3.98 \\
80 & 3200 & 0.0003000 & 4.00 & 0.0009721 & 4.00 & 0.0003093 & 4.00 & 0.0007967 & 4.00 \\
\hline
\end{tabular}
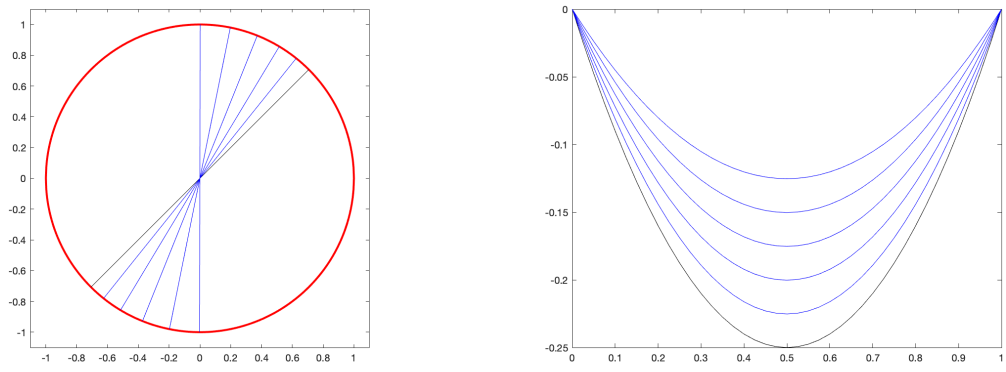

Fig. $2 \mathrm{X}^{n}$ at $t^{n}=0,0.1,0.2,0.3,0.4,0.5$ for the rotating diameter (left) and $W^{n}$ at $t^{n}=$ $0,0.1,0.2,0.3,0.4,0.5$ for the shrinking parabolic (right).

\section{References}

1. J.W. Barrett, K. Deckelnick and V. Styles: Numerical analysis for a system coupling curve evolution to reaction diffusion on the curve. SIAM Journal on Numerical Analysis, vol 55 (2017), p.1080-1100

2. J.W. Barrett, H. Garcke and R. Nürnberg: On the variational approximation of combined second and fourth order geometric evolution equations SIAM J. Scientific Comput. 29, (2007), 1006-1041.

3. J.W. Barrett, H. Garcke and R. Nürnberg: The approximation of planar curve evolutions by stable fully implicit finite element schemes that equidistribute. Numerical Methods for Partial Differential Equations, 27 (2011), pp. 1-30.

4. K. Deckelnick and G. Dziuk: On the approximation of the curve shortening flow. Calculus of Variations, Applications and Computations, (Pitman, 1995), p.100-108

5. K. Deckelnick and C.M. Elliott: Finite element error bounds for a curve shrinking with prescribed normal contact to a fixed boundary. IMA Journal of Numerical Analysis, vol 18 (Oxford University Press, 1998), p.635-654

6. K. Deckelnick, C.M. Elliott and V. Styles: Numerical diffusion-induced grain boundary motion. Interfaces and Free Boundaries, vol 3 (1998), p.393-414

7. G. Dziuk: Convergence of a semi-discrete scheme for the curve shortening flow. Mathematical Models and Methods in Applied Sciences, vol 4 (World Scientific, 1994), p.589-606

8. C.M. Elliott and H. Fritz: On approximations of the curve shortening flow and of the mean curvature flow based on the DeTurck trick. IMA Journal of Numerical Analysis, vol 37 (2016), p.543-603

9. P. Pozzi and B. Stinner: Curve shortening flow coupled to lateral diffusion. Numerische Mathematik, vol 135 (Springer, 2017), p.1171-1205 